\title{
Lipoteichoic Acid Isolated from Lactobacillus plantarum Inhibits Melanogenesis in B16F10 Mouse Melanoma Cells
}

\author{
Hye Rim Kim ${ }^{1}$, Hangeun Kim ${ }^{1,2}$, Bong Jun Jung ${ }^{1}$, Ga Eun You ${ }^{1}$, Soojin Jang ${ }^{3}$, and Dae Kyun Chung ${ }^{1,2,4, *}$
}

\begin{abstract}
Lipoteichoic acid (LTA) is a major component of the cell wall of Gram-positive bacteria. Its effects on living organisms are different from those of lipopolysaccharide (LPS) found in Gram-negative bacteria. LTA contributes to immune regulatory effects including anti-aging. In this study, we showed that LTA isolated from Lactobacillus plantarum (pLTA) inhibited melanogenesis in B16F10 mouse melanoma cells. pLTA reduced the cellular activity of tyrosinase and the expression of tyrosinase family members in a dose-dependent manner. The expression of microphthalmia- associated transcription factor (MITF), a key factor in the synthesis of melanin, was also decreased by pLTA. Further, we showed that pLTA activated melanogenesis signaling, such as extracellular signal-regulated kinase (ERK) and phosphatidylinositol 3-kinse (PI3K)/AKT. In addition, the expression of heterogeneous nuclear ribonucleoprotein A1 (hnRNP A1) and HuR, which are important RNA-binding proteins (RBPs), was reduced. pLTA likely degrades MITF via regulation of melanogenic signaling and RNA stability of melanogenic proteins, resulting in the reduction of melanin. Thus, our data suggest that pLTA has therapeutic potential for treating hyperpigmentation disorders and can also be used as a cosmetic whitening agent.
\end{abstract}

\section{INTRODUCTION}

Melanin, the pigment responsible for the color of eyes, skin, and hair, is secreted by melanocytes in the basal layer of the dermis (Lin and Fisher, 2007). The role of melanin is to protect the skin from ultraviolet (UV) damage by absorbing UV rays and removing reactive oxygen species (ROS) (Brenner and Hearing, 2008; Shen et al., 2012).

\footnotetext{
${ }^{1}$ School of Biotechnology and Institute of Life Science and Resources, ${ }^{2}$ RNA Inc., College of Life Science, Kyung Hee University, Yongin 449701 , Korea, Institute Pasteur Korea, Seongnam 463-400, Korea, ${ }^{4}$ Skin Biotechnology Center, Gyeonggi Biocenter, Suwon 443-766, Korea *Correspondence: dkchung@khu.ac.kr
}

Received 1 October, 2014; revised 5 November, 2014; accepted 18 November, 2014; published online, 15 January, 2015

Keywords: Lactobacillus plantarum, lipoteichoic acid, melanogenesis, microphthalmia-associated transcription factor (MITF), RNA stability
Melanogenesis refers to the process of melanin synthesis. Melanogenesis is regulated by multiple enzymatic and chemical reactions in melanocytes. Enzymes responsible for the regulation of melanogenesis include tyrosinase, tyrosinaserelated protein 1 (TRP-1), and dihydroxyphenylalanine chrome tautomerase (also known as tyrosinase-related protein 2, TRP-2). Microphthalmia-associated transcription factor (MITF) plays a critical role in melanogenesis as a transcriptional activator of tyrosinase, TRP-1, and TRP-2 (Boissy, 2003; Busca et al., 1996; Costin and Hearing, 2007; Pawelek and Chakraborty, 1998). Previous studies revealed that three major signaling pathways are known to regulate melanogenesis: the protein kinase C-mediated pathway (PKC), the cyclic adenosine monophosphate (CAMP) signaling-mediated pathway, and the mitogen-activated protein kinase (MAPK) (Lee et al., 2007). Of these, CAMP, which is involved in melanogenesis via phosphatidylinositol 3-kinase (PI3K)/AKT, Wnt/ $\beta$-catenin, and GSK3 $\beta$ signaling pathways, regulates melanogenesis through expression of MITF and tyrosinase. The ERK pathway appears to influence the synthesis of melanin via a negative feedback mechanism involving cAMP (Englaro et al., 1998). Activation of PI3K/AKT signaling phosphorylates MITF, leading to negative regulation of melano-genesis (Su et al., 2013).

The production of melanin is an important defense system of the skin, but excessive accumulation of melanin induces hyper pigmentation disorders such as freckles, lentigo, and melasma (Ahn et al., 2006; lozumi et al., 1993; Li et al., 2003). There is great interest in discovering depigmentation agents to prevent or treat hyperpigmentation disorders for medical or cosmetic reasons. Several existing whitening agents have restricted uses because of side-effects or low stability. For example, kojic acid is cytotoxic and associated with side-effects such as dermatitis. Ascorbic acid has very low stability (Draelos, 2007; Kim et al., 2008c; Koo et al., 2010). An ideal whitening agent should be safe and stable as well as inhibit melanogenesis without side-effects.

Bacteria are categorized as Gram-positive or Gram-negative according to differences in the major components of their cell walls. The cell walls of the former contain lipoteichoic acid (LTA), whereas those of the latter contain lipopolysaccharide (LPS) (Issac, 2002; Morrison and Ryan, 1987; Sharma et al., 2011). Generally, LPS and LTA can also stimulate production of cytokines, such as tumor necrosis factor-alpha (TNF- $\alpha$ ) and interleukin-6 (IL-6), and excessive immune response to LPS and LTA results in severe sepsis. Unlike Staphylococcus aureus, which can cause severe sepsis (Kim et al., 2008a; 2008b; Zeng 
et al., 2010), some Gram-positive lactic acid bacteria do not induce sepsis. Probiotics are microorganisms that provide health benefit to the host. Most lactic acid bacteria are probiotics. These bacteria are found mainly in the intestine. They prevent overgrowth of pathogenic bacteria in the intestine and are also involved in immune regulation, immune boosting, and lowering the total level of cholesterol in the blood (Paravez et al., 2006). Previous studies have demonstrated that lactic acid bacteria have other beneficial effects, such as therapeutic effects on atopic dermatitis, allergies, and photo-aging. However, it is difficult to clarify the precise signaling mechanisms underlying these effects of lactic acid bacteria because various components, such as LTA, lipoproteins, and DNA all appear to be involved. In previous experiments aimed at determining the role of LTA in immune regulation, highly purified LTA was prepared from Lactobacillus plantarum (pLTA), and LTA was shown to be involved in many of the beneficial effects of this lactic acid bacterium (Kim et al., 2008a; 2008b).

Although pLTA has a variety of activities, the role of LTA on skin cells is unclear. The effect of LTA on melanogenesis is also not known. Previous studies of melanogenesis regulation by Lactobacillus focused on Lactobacillus itself or fermentation of certain substances by Lactobacillus; however, there was no focus on cell components such as LTA. Accordingly, we applied pLTA to melanocytes to clarify its effects on melanogenesis. In this study, we confirmed that pLTA inhibited melanogenesis, highlighting its potential as a promising skin-whitening agent. Furthermore, we found that pLTA-induced mRNA stability of proteins is involved in melanogenesis.

\section{MATERIALS AND METHODS}

\section{Cell lines}

B16F10 mouse melanoma cells (KCLB, Korea) were cultured in Dulbecco's modified Eagle's medium (DMEM; Welgene, Korea) supplemented with $10 \%$ heat-inactivated fetal bovine serum (FBS; Welgene), $100 \mathrm{U} / \mathrm{ml}$ of penicillin, and $100 \mu \mathrm{g} / \mathrm{ml}$ of streptomycin at $37^{\circ} \mathrm{C}$ in a humidified $5 \% \mathrm{CO}_{2}$ incubator. Cells were cultured in a $75 \mathrm{~cm}^{3}$ tissue culture flask and passaged every 2 days. pLTA treatment began $12 \mathrm{~h}$ after the cells had been seeded to ensure stabilization, and cells were stimulated with alpha-melanocyte stimulating hormone $(\alpha-M S H)$ (SigmaAldrich, USA).

\section{LTA isolation from L. plantarum}

L. plantarum (KCCM, Korea) was cultured in Man, Rogosa, and Sharpe (MRS) broth for $12 \mathrm{~h}$ at $37^{\circ} \mathrm{C}$. Cultured bacteria were harvested and suspended in $0.1 \mathrm{M}$ sodium citrate buffer $(\mathrm{pH}$ 4.7). They were subjected to ultrasonication, and disrupted cells were mixed with an equal volume of $n$-butanol for $1 \mathrm{~h}$ at room temperature, followed by centrifugation at $13,000 \times g$ for $20 \mathrm{~min}$ to obtain the aqueous phase. Aqueous phase containing LTA was evaporated and dialyzed against pyrogen-free water. LTA was first purified by hydrophobic interaction chromatography. Column fractions containing LTA were collected after a phosphate assay and then dialyzed.

\section{Cell viability assay}

Cytotoxicity of pLTA on B16F10 mouse melanoma cells was determined with a colorimetric WST-1 assay (Takara, Japan). The WST-1 assay is based on the cleavage of tetrazolium salts by mitochondrial dehydrogenases in viable cells. B16F10 cells $\left(1 \times 10^{4}\right.$ cells) were seeded on 96 -well tissue culture plates and cultured for $12 \mathrm{~h}$. The first pLTA treatment was performed with the indicated concentration for $24 \mathrm{~h}$. After cells were washed with DPBS, serum-free media was added. Then, cells were stimulated with additional pLTA for $48 \mathrm{~h}$ to examine cell cytotoxicity. Premixed WST-1 reagent $(10 \mu \mathrm{l})$ was added to $100 \mu \mathrm{l}$ of the culture medium. After $30 \mathrm{~min}$ incubation, the absorbance was measured using an ELISA reader at $420 \mathrm{~nm}$.

\section{Cell-free mushroom tyrosinase activity}

Cell-free mushroom tyrosinase activity was measured using the method of Yagi (Yagi et al., 1987) with minor modification. Briefly, $40 \mu \mathrm{l}$ of $10 \mathrm{mM}$ L-dihydroxyphenylalanine (L-DOPA) (Sigma-Aldrich), $40 \mu \mathrm{l}$ of 125 units of mushroom tyrosinase (Sigma-Aldrich), $80 \mu \mathrm{l}$ of $67 \mathrm{mM}$ sodium potassium phosphate buffer ( $\mathrm{pH} 6.8)$, and $40 \mu \mathrm{l}$ of different concentrations of pLTA were mixed. Kojic acid (Sigma-Aldrich) was used as a control. Following incubation at $37^{\circ} \mathrm{C}$ for $10 \mathrm{~min}$, the amount of dopachrome formation was determined by measuring the absorbance at 415 $\mathrm{nm}$. Inhibition of the activity of mushroom tyrosinase was indicated by a reduction in absorbance of the pLTA-treated sample versus the blank sample.

\section{Intracellular activity of tyrosinase}

Intracellular tyrosinase activity was determined by measuring dopachrome formation of L-DOPA after reaction with the cell lysate. B16F10 cells $\left(1 \times 10^{5}\right)$ were seeded on 6-well tissue culture plates. After $12 \mathrm{~h}$ incubation, cells were treated with pLTA for $24 \mathrm{~h}$. The medium was then replaced with serum-free DMEM containing pLTA and cells were further incubated for 24 h. Cells were washed twice with cold DPBS and lysed with phosphate buffer $(\mathrm{pH} 6.8)$ containing $1 \%$ Triton X-100. Cell lysates were clarified by centrifugation at $13,000 \mathrm{rpm}$ for $10 \mathrm{~min}$. Each lysate $(90 \mu \mathrm{l})$ was placed in a $96-$ well plate, and a $10 \mu \mathrm{l}$ aliquot of $2 \mathrm{mg} / \mathrm{ml} \mathrm{L}$-DOPA was then added to each well. After incubation at $37^{\circ} \mathrm{C}$ for $30 \mathrm{~min}$, the absorbance was measured at $415 \mathrm{~nm}$ using an ELISA reader.

\section{Measurement of melanin content}

Melanin content was measured as described previously with minor modification (Chung et al., 2009). B16F10 cells $\left(1 \times 10^{5}\right)$ were seeded on 6-well tissue culture plates with various concentrations of pLTA for $24 \mathrm{~h}$. The medium was then replaced with serum-free DMEM containing pLTA and $\alpha-M S H$. After incubation for $48 \mathrm{~h}$, the medium was removed, and cells were washed twice with cold DPBS. To extract intracellular melanin, cell pellets were dissolved in $1 \mathrm{~N} \mathrm{NaOH}$ at $65^{\circ} \mathrm{C}$ for $30 \mathrm{~min}$. The melanin content of the cell extracts was measured at $415 \mathrm{~nm}$ with an ELISA reader. Separately, cell pellets were photographed to ensure changes in melanin content.

\section{Western blot analysis}

Total protein content of each supernatant was determined using the Bradford assay (Bio-Rad, USA). Cell lysates $(20 \mu \mathrm{g}$ of each lysate) were mixed with loading buffer $(0.25 \mathrm{M}$ Tris- $\mathrm{HCl}$, $15 \%$ SDS, $50 \%$ glycerol, $25 \% \beta$-mercaptoethanol, $0.01 \%$ bromophenol blue) and denatured at $100^{\circ} \mathrm{C}$ for $5 \mathrm{~min}$. Denatured lysates were separated by SDS-PAGE (10\% tris-glycine gels) and electro-transferred onto a PVDF membrane (Millipore, USA) for $1 \mathrm{~h}$ at $100 \mathrm{~V}$. Membranes were blocked with $5 \%$ skim milk in Tris-buffered saline (TBS) containing $0.1 \%$ Tween 20 (TBS-T buffer) for $1 \mathrm{~h}$ at room temperature. After washing three times with TBS-T, membranes were incubated with specific primary antibodies overnight at $4^{\circ} \mathrm{C}$, followed by conjugation to horseradish peroxidase secondary antibodies for $2 \mathrm{~h}$ at room temperature. Protein bands were then visualized with ECL 
Table 1. Primer sequences used for RT-PCR

\begin{tabular}{lll}
\hline MITF & Forward & CCCGTCTCTGGAAACTTGATCG \\
& Reverse & CTGTACTCTGAGCAGGTG \\
B-actin & Forward & GTGCAATTCCTGAGCTGACA \\
& Reverse & CTTAAAGATGGCCAGCAAGC \\
\hline
\end{tabular}

Table 2. The primer sequence used for Real time-PCR

\begin{tabular}{lll}
\hline MITF & Forward & AACAAGGGAACCATTCTCAAGG \\
& Reverse & AGATCAGGCGAGCAGAGACC \\
\multirow{3}{*}{-actin } & Forward & AATCGTGCGTGACATCAAAG \\
& Reverse & GAAAAGAGCCTCAGGGCAT \\
\hline
\end{tabular}

reagents (BioNote, Korea), and expression of target proteins was normalized to that of $\beta$-actin. Commercially available antibodies to MITF, tyrosinase, hnRNP A1, HuR, PI3K p85 $\alpha$, and PI3K p110 $\beta$ (Abcam, USA); p-ERK and p-AKT (Cell Signaling, USA) $\beta$-actin and TRP-1 (Santa Cruz Biotechnology, USA); TRP-2, anti-rabbit, and anti-mouse (Amersham Biosciences, Sweden) were procured.

Reverse transcriptase-polymerase chain reaction (RT-PCR) and real-time PCR

Total RNA was isolated from B16F10 cells using RNA-Bee (TEL-TEST, USA) according to the manufacturer's instructions and quantified by measuring the absorbance at $260 \mathrm{~nm}$. RNA was then reverse-transcribed using Novel ZymoTaq Plus mix (Novel Bioscience, Korea). The reaction was cycled 28 times for $30 \mathrm{~s}$ at $94^{\circ} \mathrm{C}, 30 \mathrm{~s}$ at $57^{\circ} \mathrm{C}$, and $2 \mathrm{~min}$ at $72^{\circ} \mathrm{C}$ for MITF and 27 times for $30 \mathrm{~s}$ at $94^{\circ} \mathrm{C}, 30 \mathrm{~s}$ at $55^{\circ} \mathrm{C}$, and $1 \mathrm{~min}$ at $72^{\circ} \mathrm{C}$ for $\beta$-actin. Specific primers used for RT-PCR were designed according to the method reported by Lee et al. (2010) (Table 1). Expression of mRNA was normalized to that of $\beta$-actin. PCR products were visualized by electrophoresis on $1.5 \%$ agarose gels stained with ethidium bromide. To quantify mRNA expression, real-time PCR amplification was carried out on an $A B I$ Prism 7500 Sequence Detection system (Applied Biosystems, USA) with SYBR premix Ex TaqTM II (Takara, Japan). Expression levels of the tested genes were calculated using the delta Ct method and normalized to the expression levels of $\beta$-actin. Sequences for real-time PCR primers are listed in Table 2 (Seong et al., 2012).

\section{RNA electro mobility shift assay (RNA EMSA)}

Nuclear extracts were prepared from B16F10 cells using NEPER Nuclear and Cytoplasmic Extraction Reagents (Thermo Pierce Biotechnology Scientific, USA). RNA-EMSAs were performed using biotinylated RNA probes and the Light Shift TM Chemiluminescent EMSA kit (Thermo Pierce Biotechnology Scientific) according to the manufacturer's protocol. A biotinylated MITF mRNA probe containing the AUUUA motifs was synthesized by Bioneer corporation (Korea). Supershift was accomplished by preincubating with anti-hnRNP A1, anti-HuR (Abcam) and IgG control antibodies, IgG (Santa Cruz Biotechnology). RNA-protein complexes were separated on $5 \%$ nondenaturing polyacrylamide gels run in TBE buffer. Then, the gels were transferred to nylon membranes (GE Healthcare, UK). Transferred RNAs were crosslinked to the membrane and detected with a chemiluminescence EMSA kit (Thermo Pierce Biotech
A

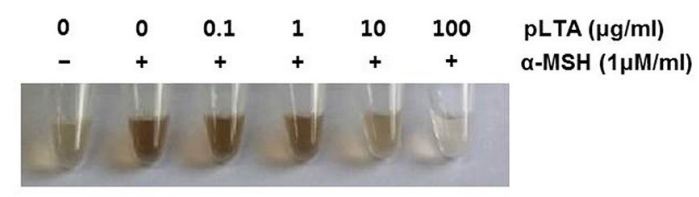

B
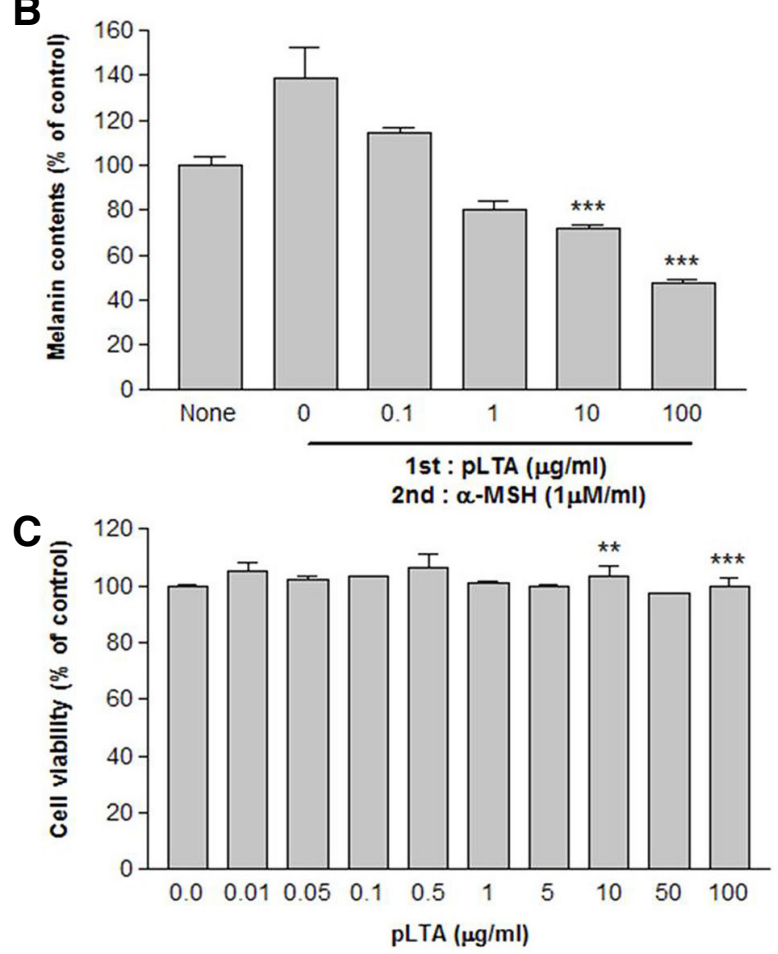

Fig. 1. Effect of pLTA on melanin synthesis in B16F10 cells. The 1 $\times 10^{5}$ B16F10 cells $(n=3)$ were incubated with the indicated concentrations of pLTA for $72 \mathrm{~h}$. Cells were dissolved in $1 \mathrm{~N} \mathrm{NaOH}$ and photographed (A). Alternatively, melanin content was determined as described in the "Materials and Methods" (B). Cell viability was determined by WST-1 assays (C). Results are the means of three independent experiments (mean \pm S.D). ${ }^{* \star \star} P<0.001$ versus $\alpha$ MSH treated cells.

nology Scientific).

\section{Immunoprecipitation assay}

Harvested cells were lysed in lysis buffer $(50 \mathrm{mM}$ Tris $\mathrm{HCl}, 150$ $\mathrm{mM} \mathrm{NaCl}, 1 \% \mathrm{NP}-40,0.5 \%$ sodium deoxylcholate, $0.1 \%$ SDS) and preincubated with magnetic beads (Cell Signaling) for 30 $\min$ at $4^{\circ} \mathrm{C}$. Then, preincubated cells were centrifuged and supernatants were mixed with magnetic beads and anti-MITF antibody with gentle rocking overnight at $4^{\circ} \mathrm{C}$. The immune complexes were precipitated by centrifugation and washed three times with cold PBS buffer. After washing, the immune complexes were resuspended in 2X SDS loading buffer and boiled for $5 \mathrm{~min}$. Boiled samples were separated by SDSPAGE and proteins were transferred to PVDF membranes for immunoblotting.

\section{RESULTS}

pLTA decreases melanogenesis in B16F10 cells

To investigate whether pLTA inhibited melanogenesis, degradation of melanin synthesis in $\alpha-\mathrm{MSH}$-treated cells was visual- 


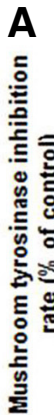

B
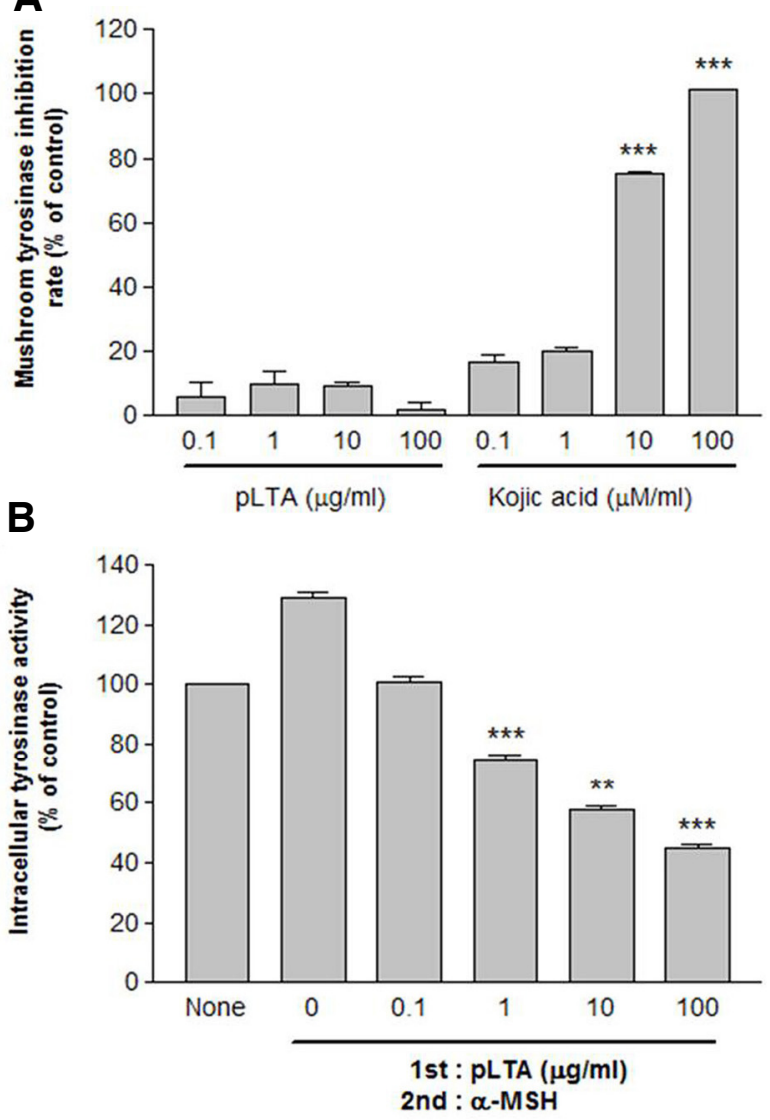

C

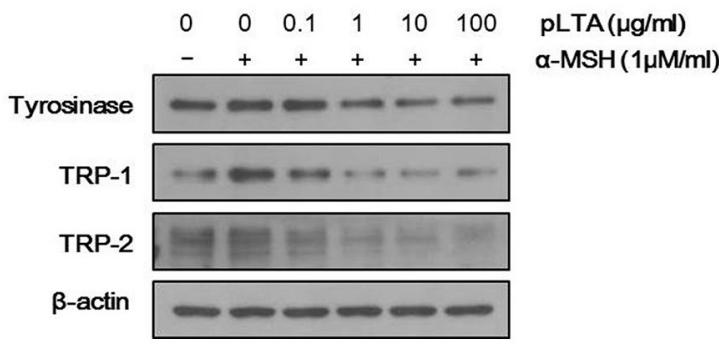

Fig. 2. Effect of pLTA on tyrosinase activity. Cell-free tyrosinase activity was determined using mushroom tyrosinase (A). Intracellular tyrosinase was measured in B16F10 cell lysates (B). Expression of tyrosinase family members including tyrosinase, TRP-1, and TRP-2 were analyzed by Western blot analysis. To verify the amount of loaded protein, the blots were also probed with anti- $\beta$ actin (C). Experiments were performed using separate stock culture $(n=3)$. Results are the means of three independent experiments (mean \pm S.D). ${ }^{* \star} P<0.01 ;{ }^{* \star *} P<0.001$ versus control (A) and $\alpha-$ MSH treated cells (B).

ized. The melanin content of B16F10 cells after pLTA treatment was measured, and cells were photographed (Fig. 1A). pLTA decreased $\alpha-\mathrm{MSH}$-mediated melanin synthesis in a dosedependent manner. Melanin content was reduced to $57.9 \%$ by treatment with $100 \mu \mathrm{g} / \mathrm{ml}$ pLTA compared to the control group treated with $\alpha-\mathrm{MSH}$ only which showed $126 \%$ melanin content (Fig. 1B). To evaluate the cytotoxicity of pLTA on B16F10 mouse
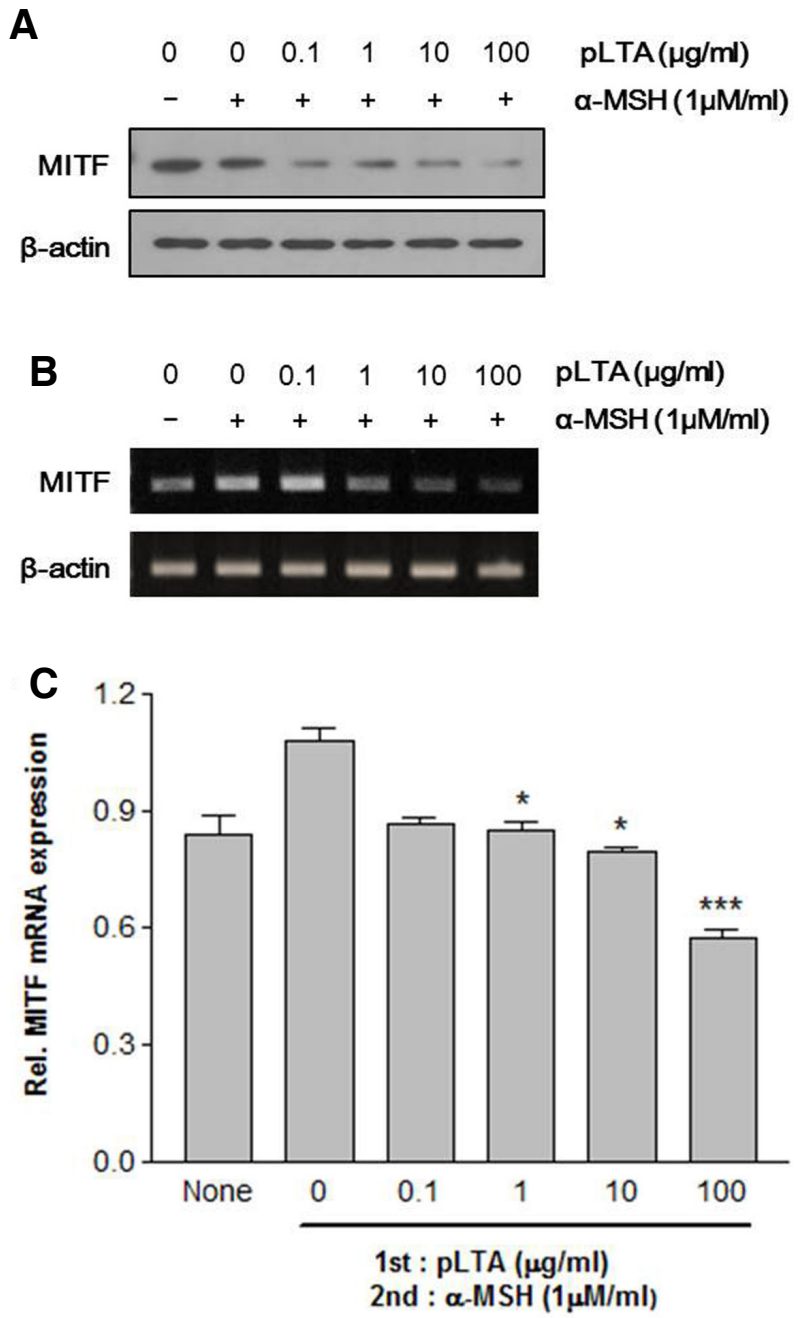

Fig. 3. Effect of pLTA on expression of MITF. The $1 \times 10^{5} \mathrm{~B} 16 \mathrm{~F} 10$ cells $(n=3)$ were incubated at the indicated concentrations of pLTA for $24 \mathrm{~h}$. After pretreatment with pLTA, cells were treated with $\alpha$ $\mathrm{MSH}$. Expression of MITF was analyzed by Western blot analysis (A) and MITF transcript levels were analyzed by RT-PCR (B) and real-time PCR $(C)$. The $\beta$-actin was used for loading $(A)$ and internal control $(\mathrm{B}, \mathrm{C})$. Data are expressed as the mean \pm S.D. (C). ${ }^{*} P<$ $0.05 ;{ }^{* * \star} P<0.001$ versus $\alpha-\mathrm{MSH}$ treated cells.

melanoma cells, we performed a WST-1 assay after treatment with pLTA for $48 \mathrm{~h}$. As shown in Fig. 1C, cell death was not observed after treatment with pLTA at a concentration range of $0.01-100 \mu \mathrm{g} / \mathrm{ml}$. This result indicates that decreased melanin synthesis in $\alpha-\mathrm{MSH}$-treated cells was not consistent with cell death. Therefore, pLTA may affect melanin synthesis.

pLTA inhibits intracellular tyrosinase activity

The activity of tyrosinase was examined in a cell-free system using mushroom tyrosinase to determine whether pLTA inhibited tyrosinase activity. We compared the melanogenic activity of pLTA with that of kojic acid, a potent inhibitor of tyrosinase activity. pLTA had no direct inhibitory effect on tyrosinase, whereas kojic acid inhibited mushroom tyrosinase activity in a dosedependent manner (Fig. 2A). We also measured the effect of 


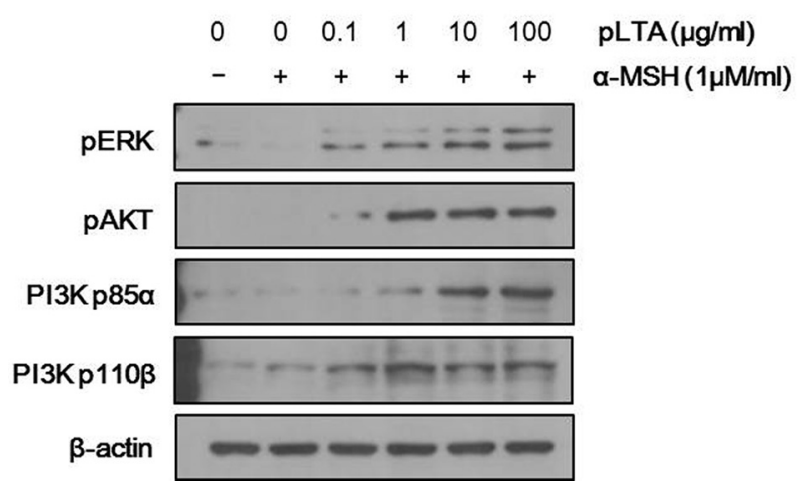

Fig. 4. Activation of ERK and PIBK/AKT signaling by pLTA stimulation. The $1 \times 10^{5} \mathrm{~B} 16 \mathrm{~F} 10$ cells $(n=3)$ were incubated at the indicated concentrations of pLTA for $24 \mathrm{~h}$, and then stimulated with $\alpha-$ $\mathrm{MSH}$ for $2 \mathrm{~h}$. Levels of $\mathrm{p}$-ERK and $\mathrm{p}$-PI3K/AKT were measured by Western blot analysis. To verify the amount of loaded protein, the blots were also probed with anti- $\beta$-actin.

pLTA on the intracellular activity of tyrosinase using B16F10 cell lysates. The results showed that 10 and $100 \mu \mathrm{g} / \mathrm{ml} \mathrm{pLTA}$ dose-dependently reduced the intracellular activity of tyrosinase to $57.6 \%$ and $44.6 \%$, respectively (Fig. 2B). We also conducted Western blot analysis to evaluate changes in protein levels of tyrosinase family members including tyrosinase, TRP-1, and TRP-2 (Fig. 2C). The $\alpha-M S H$ increased tyrosinase, TRP-1, and TRP-2 protein levels, whereas pLTA inhibited synthesis of these tyrosinase family members. The relative density of protein bands is shown after normalization with $\beta$-actin. This result suggests that pLTA inhibited melanogenesis by decreasing the activity of intracellular tyrosinase, with no direct effect on tyrosinase itself.

\section{MITF expression is inhibited by pLTA}

Next, we examined the effect of pLTA on MITF expression by Western blot analysis. MITF is a key transcription factor in melanogenesis, and expression of MITF increases transcription of members of the tyrosinase gene family such as tyrosinase, TRP-1, and TRP-2 (Boissy, 2003; Pawelek and Chakraborty, 1998). As shown in Fig. 3A, B16F10 cells treated with pLTA and $\alpha-\mathrm{MSH}$ showed decreased expression of MITF at the protein level as compared to cells treated with only $\alpha-\mathrm{MSH}$. MITF mRNA levels were also decreased in a pLTA dose-dependent manner (Fig. 3B). Quantitative real-time PCR results showed that pLTA inhibited about $40 \%$ of MITF mRNA compared to $\alpha$ MSH-treated cells (Fig. 3C). The reduction of MITF by pLTA was consistent with the inhibition of tyrosinase family proteins. These results indicate that pLTA inhibits expression of MITF, which in turn triggers a decrease in expression of tyrosinase, TRP-1, and TRP-2

\section{pLTA activated ERK and PI3K/AKT signaling pathway}

Studies have shown that signaling by MAPK and PI3K/AKT suppresses melanogenesis through the degradation of MITF (Boissy, 2003; Busca et al., 1996; Lee et al., 2007). Thus, to further investigate the effect of pLTA on melanogenesis, we examined protein expression levels of pERK and PI3K/AKT. As shown in Fig. 4, the phosphorylation of ERK and AKT as well as PI3K subunits, such as $p 85 \alpha$ and $p 110 \beta$, significantly increased after pLTA treatment in a dose-dependent manner in $\alpha-\mathrm{MSH}$-treated cells. These results indicate that MITF can be

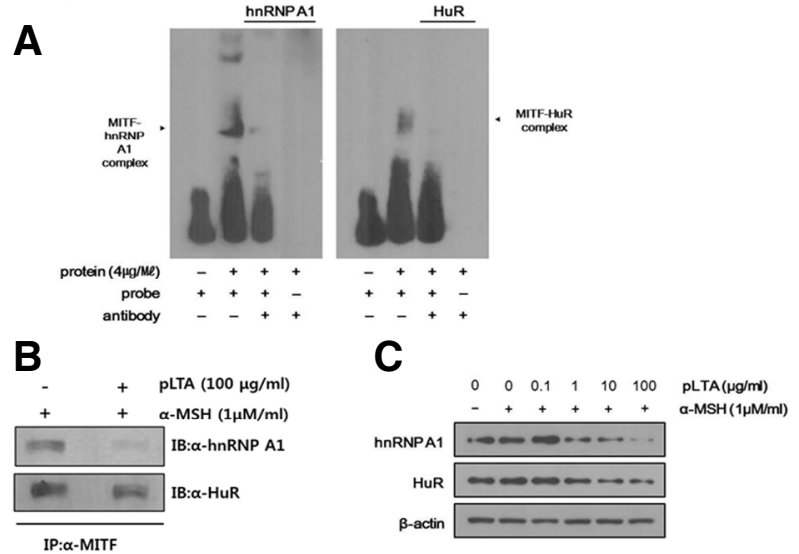

Fig. 5. Interaction between RBPs and MITF and effect of pLTA on the mRNA stability of melanogenic proteins. The $1 \times 10^{5}$ B16F10 cells $(n=3)$ were incubated with $\alpha-\mathrm{MSH}$ for $48 \mathrm{~h}$ in the presence or absence of pLTA. RNA EMSA was conducted with nuclear extracts containing protein and $10 \mathrm{fmol}$ of biotinylated probe. Anti-hnRNP A1 and anti-HuR antibodies were added to the reaction. The mixture was separated by electrophoresis and detected by immunoblot analysis with anti-hnRNP A1 or anti-HuR antibody. Arrows indicate the position of the hnRNP A1/HuR band (A). Immunoprecipitation assays were performed with anti-MITF antibody for identification of hnRNP A1 or HuR-MITF complexes. Precipitated proteins were analyzed by immunoblot assays using anti-hnRNP A1 or anti-HuR antibody (B). In order to clarify the influence of pLTA, the protein levels of hnRNP A1 and HuR after pLTA treatment were measured by Western blot analysis. To verify the amount of loaded protein, the blots were probed with anti- $\beta$-actin (C).

activated by ERK and PI3K/AKT signaling in pLTA-treated cells.

PLTA reduces melanogenesis by affecting the stability of MITF RNA

RNA-binding proteins (RBPs) play an important role in gene regulation by affecting RNA stability and translation. hnRNPs and ELAV/Hu proteins are representative groups of RBPs (Papadopoulou et al., 2013). First, we performed a RNA Electro Mobility Shift Assay (RNA EMSA) to investigate whether hnRNP A1 and HuR interact with ARE on the 3'-UTR of MITF. The RNA EMSA was performed on nuclear extracts containing $4 \mathrm{ug} / \mathrm{ml}$ of total protein from B16F10 cells treated with a biotinylated MITF probe. Then anti-hnRNP A1, HuR, and control antibody ( $\mathrm{IgG}$ ) were added to the mixtures for the supershift assay. As shown in Fig. 5A, shifted bands were detected indicating that hnRNP A1 and HuR interact with the 3'-UTR of MITF. No shift was observed with the free probe (Lane 1) or the complex with free anti-lgG as a negative control (data were not shown). Disappearance of the RNA-protein complex in the supershift assay with anti-hnRNP or -HuR antibody provides evidence that the RNA-protein complex was supershifted by specific antibody. These results indicate that hnRNP A1 and HuR specifically bind to MITF mRNA. Binding of hnRNP A1 and HuR to MITF was reconfirmed by an immunoprecipitation assay. As shown in Fig. 5B, specific bands were detected in the anticipated complexes after immunoblotting using anti-hnRNP A1 or anti-HuR antibodies. The protein complexes were reduced in pLTA-treated cells compared to cells treated with $\alpha-\mathrm{MSH}$ only. These results indicate MITF mRNA is degraded after treatment with pLTA. To determine whether the inhibitory effect of pLTA 
on melanogenesis was linked to its effects on the stability of MITF RNA, we examined the expression level of RBPs after pLTA treatment. The expression of hnRNP A1 and HuR was dramatically reduced in pLTA-treated cells compared to cells treated with $\alpha-\mathrm{MSH}$ only (Fig. $5 \mathrm{C}$ ). This result suggests that pLTA affects not only melanogenic signaling pathways, but also the RNA stability of MITF, a transcription factor of melanogenesis.

\section{DISCUSSION}

Melanocytes protect the body from UV radiation by producing melanin and eliminating reactive oxygen species (ROS). However, abnormal accumulation of melanin causes esthetic problems and hyperpigmentation disorders, such as freckles, chloasma, lentigo, liver spots, and melasma (Ahn et al., 2006; lozumi et al., 1993; Li et al., 2003; Unver et al., 2006). Arbutin, kojic acid, hydroquinone, and ascorbic acid have been used to treat hyperpigmentation disorders. However, the use of these agents is limited due to their side-effects or instability (Draelos, 2007; Koo et al., 2010).

Prior studies showed that LTA from L. plantarum has many beneficial effects, such as intestinal regulation, immune system regulation, skin moisturizing, and anti-photo aging. However, the influence of pLTA on melanogenesis has not been investigated previously. Thus, our aim in this study was to elucidate whether pLTA has the potential to be used for treatment of hyperpigmentation disorders and as a whitening reagent. In this study, we investigated the effects of PLTA on the inhibition of melanogenesis in B16F10 mouse melanoma cells. We evaluated the cell cytotoxicity of pLTA and found that pLTA was not cytotoxic to B16F10 mouse melanoma cells (Fig. 1), indicating that it could potentially be used as a therapeutic reagent. Generally, whitening reagents inhibit melanogenesis by inhibiting the catalytic activity of tyrosinase. Thus, we measured the concentration of melanin and the activity of tyrosinase after treatment of cells with pLTA. Melanin production was significantly reduced by pLTA. Interestingly, pLTA reduced the activity of cellular tyrosinase but had no effect on mushroom tyrosinase (Fig. 2). These findings suggest that the inhibitory effects of pLTA on melanogenesis were not due to its direct effects on tyrosinase but rather indirect regulation of the expression of tyrosinase. Therefore, we focused on determining the effect of pLTA on the melanin synthesis pathway. We showed that pLTA activated ERK and PI3K/AKT through Western blot analysis (Fig. 4). Melanogenesis is regulated by three different signaling pathways: i) the protein kinase C-mediated pathway, ii) the cAMP-mediated pathway, and iii) the mitogen-activated protein kinase (MAPK) pathway. MAPK activates MITF via phosphorylation of MITF at serine 73. However, phosphorylation of MITF at serine 73 is also essential for ubiquitination and degradation of MITF. Activation of ERK and the resulting phosphorylation of MITF at serine 73 could ultimately lead to the degradation of MITF (Hemesath et al., 1998; Xu et al., 2000). cAMP could be the starting point of several interacting signaling cascades in melanin synthesis that regulate the production of melanin through activation of PKA and phosphorylation of CREB. In melanocytes, cAMP also regulates PI3K, which consists of a regulatory subunit, $\mathrm{p} 85$, and a catalytic subunit, $\mathrm{p} 110$. Stimulation with CAMP inhibits PI3K signaling. Inhibition of PI3K increases the synthesis of melanin through increased transcription of tyrosinase and TRP-1 due to increased expression of MITF. AKT (also known as protein kinase B) is a typical effector of PI3K. Therefore, activation of ERK and PI3K/AKT signaling reduces melanogenesis via down-regulation of MITF expression (Bertolotto et al., 1998; Hennessy et al., 2005; Meinkoth et al., 1991).

Changes in the levels of MITF observed by Western blot analysis and PCR revealed that pLTA reduced the expression of MITF in a dose-dependent manner. A decrease in the expression of tyrosinase, TRP-1, and TRP-2 upon pLTA treatment was also observed by Western blot analysis (Fig. 3). The tyrosinase gene family responsible for the regulation of melanogenesis consists of tyrosinase, TRP-1, and TRP-2. Tyrosinase is a key enzyme involved in melanogenesis and catalyzes the hydroxylation of L-tyrosinase to DOPA and the oxidation of DOPA to DOPA quinone (Koo et al., 2010; Park et al., 2011). TRP-1 and TRP-2 are enzymes that belong to the tyrosinase family and regulate melanogenesis. They are involved in stabilizing tyrosinase and modulating its catalytic activity. In our, pLTA activated anti-melanogenesis signals, resulting in downregulation of MITF (Fig. 3). Down-regulation of MITF then resulted in decreased expression of tyrosinase family members and their activities, finally reducing the production of melanin. Moreover, we performed additional experiments to determine other mechanisms underlying the effect of pLTA on melanogenesis.

Regulation of mRNA is fundamental to processes such as mRNA stability, transport, translation, and localization and also important for gene expression. These processes are controlled by RBPs that mainly bind to specific elements situated in the untranslated regions (UTRs) of mRNAs. Particularly, the general mechanisms that regulate the stability of mRNA via interactions between sequence-specific RBPs and AU-rich elements (ARE). AREs, located in the 3'-UTR, are critical regulatory motifs and have overlapping AUUUA sequences. These sites are a target for RBPs, which stabilize or destabilize mRNA containing these elements. RBPs have an established role in regulating the stability and translation of target mRNA that contain ARE (Chen et al., 2002; Papadopoulou et al., 2010). Heterogeneous nuclear ribonucleoproteins (hnRNPs) and embryonic lethal abnormal vision (ELAV) proteins are representative groups of RBPs that modulate gene regulation of AREcontaining mRNA. hnRNPs include at least 20 discrete hnRNPs that are labeled as hnRNP A through $U$. These hnRNPs are actively involved in many facets of mRNA metabolism, from transcription to post-transcriptional events and translation (Papadopoulou et al., 2013; Wen et al., 2010). ELAV is another important small family of RBPs that comprises four members: $\mathrm{HuR}, \mathrm{HuB}, \mathrm{HuC}$, and HuD. ELAV proteins play a role in a variety of other biological processes, such as mRNA nuclear splicing as wells as export and stability/translation of target mRNAs. Both hnRNP A1 and HuR are mainly localized in the nucleoplasm. They regulate the stability and translation of target AREcontaining mRNAs and can shuttle between the nucleus and cytoplasm (Brennan and Steiz, 2001; Papadopoulou et al., 2013). In this study, we focused on hnRNP A1 and HuR which are mRNA stabilizing factors that stabilize ARE-containing mRNAs. The molecular size of these two proteins is close, and the interaction between hnRNP A1 and HuR has been studied in previous reports (Papadopoulou et al., 2010). MITF also contains an ARE motif in its mRNA sequence; therefore, we anticipate that degradation of MITF $m R N A$ is relative to destabilization of mRNA and RBPs. The results of RNA EMSA and immunoprecipitation experiments in our study verified the interaction between hnRNP A1/HuR and MITF. The RNA EMSA results showed complex bands of hnRNP A1/HuR and MITF mRNA, and supershift assays confirmed the disappearance of these 
complex bands by supershift. To further clarify the association of hnRNP A1/HuR and MITF, we performed immunoprecipitation assays. The results proved that hnRNP/ HuR binds to MITF. Notably, the bands were diminished in pLTA-treated samples. Western blot analysis revealed that levels of hnRNP A1 and HuR were decreased by pLTA in a dose-dependent manner. However, pLTA had no influence on cell viability. These results indicate that pLTA may have adverse effects on the mRNA stability of melanogenic proteins such as MITF, tyrosinase, TRP-1, and TRP-2, leading to inhibition of melanogenesis. However, it is our provisional opinion that the disappearance of specific bands was caused by the results of supershift assays. Thus, further studies are required to elucidate the nature of the relationship between PLTA and RNA stability in melanogenesis.

In summary, we demonstrated that pLTA has an anti-melanogenic effect. In addition, we also determined the mechanisms underlying its inhibition of melanogenesis. Specifically, we found that pLTA suppresses the expression of MITF by activating ERK and PI3K/AKT pathways and decreasing the cellular activity of tyrosinase and expression of melanogenic enzymes. We also found that pLTA treatment reduced the RNA stability of melanogenic proteins. Our results suggest that pLTA can be used as a potentially potent and safe therapeutic reagent for treatment of hyperpigmentation disorders and a whitening agent for cosmetics.

\section{ACKNOWLEDGMENTS}

This work was supported by a grant from the Next-Generate BioGreen 21 Program (No. PJ009009), Rural Development Administration, Republic of Korea.

\section{REFERENCES}

Ahn, S.J., Koketsu, M., Ishihara, H., Lee, S.M., Ha SK, Lee KH, Kang, T.H., and Kima, S.Y. (2006). Regulation of melanin synthesis by selenium-containing carbohydrates. Chem. Pharm. Bull. 54, 281-286.

Bertolotto, C., Abbe, P., Hemesath, T.J., Bille, K., Fisher, D.E., Ortonne, J.P., and Ballotti, R. (1998). Microphthalmia gene product as a signal transducer in CAMP-induced differentiation of melanocytes. J. Cell Biol. 142, 827-835.

Boissy, R.E. (2003). Melanosome transfer to and translocation in the keratinocyte. Exp. Dermatol. 12, 5-12.

Brennan, C.M., and Steitz, J.A. (2001). HuR and mRNA stability. Cell. Mol. Life Sci. 58, 266-277.

Brenner, M., and Hearing, V.J. (2008). The protective role of melanin against UV damage in human skin. Photochem. Photobiol. $84,539-549$.

Busca, R., Bertolotto, C., Ortonne, J.P., and Ballotti, R. (1996). Inhibition of the phosphatidylinositol 3-kinase/p70(S6)-kinase pathway induces B16 melanoma cell differentiation. J. Biol. Chem. 271, 31824-31830.

Chen, C.Y., Xu, N., and Shyu, A.B. (2002). Highly selective actions of HuR in antagonizing AU-rich element-mediated mRNA destabilization. Mol. Cell. Biol. 22, 7268-7278.

Chung, S.Y., Seo, Y.K., Park, J.M., Seo, M.J., Park, J.K., Kim, J.W., and Park, C.S. (2009). Fermented rice bran downregulates MITF expression and leads to inhibition of alpha-MSH-induced melano-genesis in B16F1 melanoma. Biosci. Biotechnol. Biochem. 73, 1704-1710.

Costin, G.E., and Hearing, V.J. (2007). Human skin pigmentation: melanocytes modulate skin color in response to stress. FASEB J. 21, 976-994.

Draelos, Z.D. (2007). Skin lightening preparations and the hydroquinone controversy. Dermatol. Ther. 20, 308-313.

Englaro, W., Bertolotto, C., Busca, R., Brunet, A., Pages, G., Ortonne, J.P., and Ballotti, R. (1998). Inhibition of the mitogenactivated protein kinase pathway triggers B16 melanoma cell differentiation. J. Biol. Chem. 273, 9966-9970.
Hemesath, T.J., Price, E.R., Takemoto, C., Badalian, T., and Fisher, D.E. (1998). MAP kinase links the transcription factor Microphthalmia to c-Kit signalling in melanocytes. Nature 391, 298-301.

Hennessy, B.T., Smith, D.L., Ram, P.T., Lu, Y., and Mills, G.B. (2005). Exploiting the PI3K/AKT pathway for cancer drug discovery. Nat. Rev. Drug Discov. 4, 988-1004.

lozumi, K., Hoganson, G.E., Pennella, R., Everett, M.A., and Fuller, B.B. (1993). Role of tyrosinase as the determinant of pigmentation in cultured human melanocytes. J. Invest. Dermatol. 100, 806-811.

Isaac, G. (2002). Role of lipoteichoic acid in infection and inflammation. LANCET Infect. Dis. 2, 171-179.

Kim, H.G., Kim, N.R., Gim, M.G., Lee, J.M., Lee, S.Y., Ko, M.Y. Kim, J.Y., Han, S.H., and Chung, D.K. (2008a). Lipoteichoic acid isolated from Lactobacillus plantarum inhibits lipopolysaccharideinduced TNF-a production in THP-1 cells and endotoxin shock in mice. J. Immunol. 180, 2553-2561.

Kim, H.G., Lee, S.Y., Kim, N.R., Ko, M.Y., Lee, J.M., Yi, T.H, Chung, S.K., and Chung, D.K. (2008b). Inhibitory Effects of Lactobacillus plantarum Lipoteichoic Acid (LTA) on Staphylococcus aureus LTA-Induced Tumor Necrosis Factor-Alpha Production. J. Microbiol. Biotechnol. 18, 1191-1196.

Kim, S., Jung, S.H., and Cho, C.W. (2008c). Physicochemical studies of a newly synthesized molecule, 6-methyl-3-phenethyl-3,4dihydro-1H-quinazoline-2-thione (JSH18) for topical formulations. Arch. Pharm. Res. 31, 1363-1368.

Koo, J.H., Lee, I., Yun, S.K., Kim, H.U., Park, B.H., and Park, J.W (2010). Saponified evening primrose oil reduces melanogenesis in B16 melanoma cells and reduces UV-induced skin pigmentation in humans. Lipids 45, 401-407.

Lee, J., Jung, K., Kim, Y.S., and Park, D. (2007). Diosgenin inhibits melanogenesis through the activation of phosphatidylinositol-3kinase pathway (PI3K) signaling. Life Sci. 81, 249-254.

Lee, J.E., Kim, S.Y., Jeong, Y.M., Yun, H.Y., Baek, K.J., Kwon, N.S. Park, K.C., and Kim, D.S. (2010). The regulatory mechanism of melanogenesis by FTY720, a sphingolipid analogue. Exp. Dermatol. 20, 237-241.

Li, G., Ju, H.K., Chang, H.W., Jahng, Y., Lee, S.H., and Son, J.K. (2003). Melanin biosynthesis inhibitors from the bark of Machilus thunbergii. Biol. Pharm. Bull. 26, 1039-1041.

Lin, J.Y., and Fisher, D.E. (2007). Melanocyte biology and skin pigmentation. Nature $445,843-850$.

Meinkoth, J.L., Montminy, M.R., Fink, J.S., and Feramisco, J.R. (1991). Induction of a cyclic AMP-responsive gene in living cells requires the nuclear factor CREB. Mol. Cell. Biol. 11, 1759-1764.

Morrison, D.C., Ryan, J.L. (1987). Endotoxins and disease mechanisms. Annu. Rev. Med. 38, 417-432.

Papadopoulou, C., Patrinou-Georgoula, M., and Guialis, A. (2010). Extensive association of HuR with hnRNP proteins within immunoselected hnRNP and mRNP complexes. Biochim. Biophys. Acta 1804, 692-703.

Papadopoulou, C., Ganou, V., Patrinou-Georgoula, M., and Guialis, A. (2013). HuR-hnRNP interactions and the effect of cellular stress. Mol. Cell 372, 137-147.

Park, S.Y., Jin, M.L., Kim, Y.H., Kim, Y., and Lee, S.J. (2011). Aromatic-turmerone inhibits $\alpha$-MSH and IBMX-induced melanogenesis by inactivating CREB and MITF signaling pathways. Arch. Dermatol. Res. 303, 737-744.

Parvez, S., Malik, K.A., Ah Kang, S., and Kim, H.Y. (2006). Probiotics and their fermented food products are beneficial for health. $J$ Appl. Microbiol. 100, 1171-1185.

Pawelek, J.M., and Chakraborty, A.K. (1998). The enzymology of melanogenesis. (Oxford University Press), pp. 391-400.

Seong, I., Min, H.J., Lee, J.H., Yeo, C.Y., Kang, D.M., Oh, E.S., Hwang, E.S., and Kim, J. (2012). Sox10 controls migration of B16F10 melanoma cells through multiple regulatory target genes. PLoS One 7, e31477.

Sharma, P., Dube, D., Singh, A., Mishra, B., Singh, N., Sinha, M., Dey, S., Kaur, P., Mitra, D.K., et al. (2011). Structural basis of recognition of pathogen-associated molecular patterns and inhibition of proinflammatory cytokines by camel peptidoglycan recognition protein. J. Biol. Chem. 286, 16208-16217.

Shen, T., Heo, S.I., and Wang, M.H. (2012). Involvement of the p38 MAPK and ERK signaling pathway in the anti-melanogenic effect of methyl 3,5-dicaffeoyl quinate in B16F10 mouse melanoma cells. Chem. Biol. Interact. 199, 106-111. 
Su, T.R., Lin, J.J., Tsai, C.C., Huang, T.K., Yang, Z.Y., Wu, M.O., Zheng, Y.Q., Su, C.C., and Wu, Y.J. (2013). I Involvement of the p38 MAPK and ERK signaling pathway in the anti-melanogenic effect of methyl 3,5-dicaffeoyl quinate in B16F10 mouse melanoma cells. Int. J. Mol. Sci. 14, 20443-20458.

Unver, N., Freyschmidt-Paul, P., Horster, S., Wenck, H., Stab, F., Blatt, T., and Elsasser, H.P. (2006). Alterations in the epidermaldermal melanin axis and factor XIlla melanophages in senile lentigo and ageing skin. Br. J. Dermatol. 155, 119-128.

Wen, F., Shen, A., Shanas, R., Bhattacharyya, A., Lian, F., Hostetter, G., and Shi, J. (2010). Higher expression of the heter- ogeneous nuclear ribonucleoprotein $\mathrm{k}$ in melanoma. Ann. Surg. Oncol. 17, 2619-2627.
Xu, W., Gong, L., Haddad, M.M., Bischof, O., Campisi, J., Yeh, E.T., and Medrano, E.E. (2000). Regulation of microphthalmiaassociated transcription factor MITF protein levels by association with the ubiquitin-conjugating enzyme hUBC9. Exp. Cell Res. 255, 135-143.

Yagi, A., Kanbara, T., and Morinobu, N. (1987). Inhibition of mushroom-tyrosinase by aloe extract. Planta Med. 53, 517-519.

Zeng, R.Z., Kim, H.G., Kim, N.R., Lee, H.Y., Jung, B.J., Ko, M.Y., Lee, S.Y., and Chung, D.K. (2010). Protein expression changes in human monocytic THP-1 cells treated with lipoteichoic acid from Lactobacillus plantarum and Staphylococcus aureus. Mol. Cells 6, 585-594. 